\title{
Gas-liquid chromatographic analysis of synovial fluid: volatile short-chain fatty acids in septic arthritis
}

\author{
DAVID G. BORENSTEIN, CHRISTINA A. GIBBS, AND ROBERT P. JACOBS \\ From the Division of Rheumatology, George Washington University Medical Center, Washington DC, USA
}

SUMmARY Volatile short-chain fatty acids (SCFA) in synovial fluid from 80 patients were quantified by gas-liquid chromatography (GLC). Characteristic patterns of volatile SCFA could not be associated with septic, nonseptic inflammatory, or noninflammatory groups. Mean concentrations of pentanoic and hexanoic acids were similar in all groups studied. In the septic arthritis group 3 of 4 patients with acetic acid and of 3 of 3 with 3-methyl butanoic acid had culture-proved staphylococcal infections. In patients with synovial fluid findings consistent with septic arthritis, including markedly raised leucocyte count, decreased glucose level, or detectable succinic acid, the detection of acetic and 3-methyl butanoic acids by GLC analysis may increase the suspicion that Staphylococcus aureus is the cause of the septic arthritis.

Early diagnosis and treatment of septic arthritis are important in preventing joint destruction. Septic arthritis is suspected on clinical grounds and confirmed by the demonstration of organisms on smear or culture. While simple and readily available, bacteriological tests may fail to yield a positive diagnosis due primarily to inadequate culture technique or prior antibiotic therapy. ${ }^{1}$ Other synovial fluid markers of infection such as a markedly elevated leucocyte count and decreased glucose are helpful, but not specific for establishing a diagnosis of septic arthritis. Therefore additional diagnostic methods which can differentiate septic from nonseptic synovial fluid would be useful.

Gas-liquid chromatography (GLC) is a rapid and sensitive method for the identification and quantification of bacterial metabolites in body fluids. GLC studies $^{23}$ of synovial fluid suggested the utility of an increased lactic acid concentration in the diagnosis of nongonococcal septic arthritis. Lactic acid concentrations were not consistently elevated in patients with gonococcol arthritis. We have shown that the presence of detectable succinic acid, a nonvolatile dicarboxilic acid, in synovial fluid correlated with a diagnosis of septic arthritis caused by Gram-positive and Gram-negative organisms, including the gonococcus. ${ }^{4}$ Gonococcal arthritis was not associated with an

Accepted for publication $12 \mathrm{July} 1982$.

Correspondence to David G. Borenstein, MD, George Washington University Medical Center, 2150 Pennsylvania Avenue, NW Washington, DC 20037, USA. increased concentration of lactic acid in our study. Brook et al. $^{5}$ suggested that the determination of volatile short-chain fatty acids (SCFA) in synovial fluid with a normal lactic acid concentration might be of value in the rapid diagnosis of gonococcal arthritis. They identified chromatographic peaks which appear to correspond to pentanoic and hexanoic acids in increased concentrations in synovial fluid from patients with gonococcal arthritis; both pentanoic and hexanoic acid and an increased concentration of lactic acid were detected in patients with nongonococcal septic arthritis.

We have studied by GLC synovial fluids from 80 patients with a variety of septic and nonseptic joint diseases in an attempt to confirm these data regarding volatile SCFA and to identify and quantify additional volatile SCFA. Characteristic patterns of volatile SCFA were sought which might differentiate patients with septic arthritis from those with nonseptic inflammatory arthritides.

\section{Patients and methods}

Eighty synovial fluids from 80 patients examined between July 1978 and December 1980 were studied. Patients were classified by clinical criteria into 3 groups: septic, nonseptic inflammatory, and nonseptic noninflammatory. Table 1 lists the diagnosis of these patients.

Septic arthritis was confirmed by positive synovial fluid culture (16), positive genitourinary tract culture for Neisseria gonorrhoeae (3), positive Gram stain 
Table 1 Patient groups

\begin{tabular}{|c|c|c|c|}
\hline Septic & & & 23 \\
\hline Gram-positive & & 14 & \\
\hline Staphylococcus aureus & 8 & & \\
\hline Streptococcus pyogenes & 2 & & \\
\hline Streptococcus group B & 1 & & \\
\hline Streptococcus pneumoniae & 1 & & \\
\hline Gram stain only (coccal forms) & 1 & & \\
\hline Antibiotic response only & 1 & & \\
\hline Gram-negative & & 2 & \\
\hline Proteus morganii & 1 & & \\
\hline Gram stain only & 1 & & \\
\hline Neisseria gonorrhoeae & & 7 & \\
\hline Synovial fluid & 3 & & \\
\hline Genitourinary tract & 3 & & \\
\hline Antibiotic response only & 1 & & \\
\hline Nonseptic inflammatory & & & 39 \\
\hline Gout & & 11 & \\
\hline Rheumatoid arthritis & & 10 & \\
\hline Reiter's syndrome & & 7 & \\
\hline Pseudogout & & 3 & \\
\hline Psoriatic arthritis & & 2 & \\
\hline Miscellaneous & & 6 & \\
\hline Inflammatory polyarthritis & 4 & & \\
\hline Inflammatory bowel disease & 1 & & \\
\hline Polyarteritis nodosa & 1 & & \\
\hline Nonseptic noninflammatory & & & 18 \\
\hline $\begin{array}{l}\text { Osteoarthritis } \\
\text { Trauma }\end{array}$ & & $\begin{array}{r}13 \\
5\end{array}$ & \\
\hline Total patients & & & 80 \\
\hline
\end{tabular}

and response to antibiotics (2), or response to antibiotics alone (2). Fourteen septic synovial fluids were obtained prior to antibiotic therapy. In 9 patients, 4 with Gram-positive, 1 with Gram-negative, and 4 with $N$. gonorrhoeae infection, synovial fluids were obtained 1 to 9 days after the start of antibiotic therapy. When multiple synovial fluid samples were available, only the initial fluid was included in this report. Primary diagnoses of patients in the septic arthritis group included rheumatoid arthritis (6), alcoholism (3), diabetes mellitus (2), systemic lupus erythematosus (1), and osteoarthritis (1).

All arthrocenteses were performed for diagnostic or therapeutic reasons. All fluids were cultured in hospital clinical microbiology laboratories. Cell counts, crystal analysis, and protein and glucose determinations were done on remaining fluid, when available.

\section{EXTRACTION PROCEDURE}

Synovial fluids were centrifuged at $500 \mathrm{~g}$ at $4^{\circ} \mathrm{C}$ for 10 minutes. Supernatant fluids were removed and were analysed immediately or stored at $-70^{\circ} \mathrm{C}$. The extraction procedure was a modification of the VPI Anaerobic Laboratory Manual ${ }^{6}$ method for extracting volatile SCFA. In brief, $0.1 \mathrm{mg}$ internal standard octanoic acid, $0.3 \mathrm{ml} 10 \mathrm{~N}$ sulphuric acid, $0.4 \mathrm{~g}$ sodium chloride, and $1 \mathrm{ml}$ ethyl ether were added to 1 $\mathrm{ml}$ of cell-free supernatant fluid. The tube was agitated with gentle inversion for 1 minute. The mixture was centrifuged at $1500 \mathrm{~g}$ at room temperature for 2 minutes to separate ether and fluid layers. A $3 \mu l$ sample was taken from the top (ether) layer and analysed by GLC.

\section{GLC ANALYSIS}

A Hewlett Packard 5730A gas chromatograph (Hewlett Packard Co., Avondale, Pennsylvania) equipped with a flame ionisation detector was employed in these studies. A 5 foot $(1.5 \mathrm{~m}) \times 4 \mathrm{~mm}$ internal diameter glass column was packed with $15 \%$ SP $1220 / 1 \%$ H3P04 on $100 / 120$ Chromosorb W AW. Routine analysis conditions were injection block $250^{\circ} \mathrm{C}$, detector port $300^{\circ} \mathrm{C}$, oven $125^{\circ} \mathrm{C}$ for 4 minutes increasing $4^{\circ} \mathrm{C}$ every 4 minutes to a maximum of $160^{\circ} \mathrm{C}$. Air, hydrogen, and nitrogen flows were 240,60 , and $60 \mathrm{ml} / \mathrm{min}$, respectively. Hewlett Packard 3380A integrator at slope sensitivity 0.03 recorded retention times and absolute amounts of SCFA compared with the internal standard. A standard mixture of volatile SCFA including acetic, propanoic, 2-methyl propanoic, butanoic, 3-methyl butanoic, pentanoic, 4-methyl pentanoic, hexanoic (Supelco Co., Bellafonte, Pennsylvania), and internal standard (heptanoic acid) were used to identify retention times and quantitation of fatty acids prior to clinical sample determinations.

\section{STATISTICAL ANALYSIS}

All statistical analyses were conducted with the data management and analysis capabilities of the Statistical Analyses System (SAS).

For all analyses the absence of an acid is treated as a zero data point instead of the weaker option of omitting the patient from the particular analysis.

The mean levels of 3 groups were analysed by a least-squares analysis of variance for unbalanced data, that is, data with unequal sample sizes in each group. The means were tested by pairwise $t$ tests when the overall (3-group) comparison reached a significance level of $0 \cdot 05$.

\section{Results}

Volatile short-chain fatty acids identified in the 80 synovial fluids are listed in Table 2 . A majority of noninflammatory fluids contained pentanoic and hexanoic acids, while acetic acid was detected infrequently. Propanoic, 2-methyl propanoic, butanoic, 3-methyl butanoic, and 4-methyl pentanoic acids 
Table 2 Volatile short-chain fatty acids in synovial fluid

\begin{tabular}{|c|c|c|c|c|c|c|c|c|}
\hline & Acetic & Propanoic & $\begin{array}{l}\text { 2-Methyl } \\
\text { propanoic }\end{array}$ & Butanoic & $\begin{array}{l}\text { 3-Methyl } \\
\text { butanoic }\end{array}$ & Pentanoic & $\begin{array}{l}\text { 4-Methyl } \\
\text { pentanoic }\end{array}$ & Hexanoic \\
\hline \multicolumn{9}{|l|}{ Noninflammatory $(n=18)$} \\
\hline Number with acid (\%) & $3(17)$ & $0(0)$ & $0(0)$ & $0(0)$ & $0(0)$ & $17(94)$ & $0(0)$ & $15(83)$ \\
\hline Range (mg/dl) & $0 \cdot 0-1 \cdot 5$ & 0 & 0 & 0 & 0 & $0.0-0.89$ & $\mathbf{0}$ & $0.0-0.47$ \\
\hline \multicolumn{4}{|l|}{ Inflammatory $(n=39)$} & 0 & 0 & $0 \cdot 33$ & 0 & $0 \cdot 20$ \\
\hline Number with acid (\%) & $3(7)$ & $6(15)$ & $0(0)$ & $0(0)$ & $0(0)$ & $30(77)$ & $0(0)$ & $32(82)$ \\
\hline Range (mg/dl) & $0.0-3.9$ & $0.0-0.66$ & 0 & 0 & 0 & $0.0-4.9$ & 0 & $0.0-0.58$ \\
\hline Mean (mg/dl) & $0 \cdot 13$ & 0.04 & 0 & 0 & $\mathbf{0}$ & 0.68 & 0 & 0.22 \\
\hline \multicolumn{9}{|l|}{ Septic $(n=23)$} \\
\hline Number with acid (\%) & $5(22)$ & $1(4)$ & $0(0)$ & $3(13)$ & $3(13)$ & $16(70)$ & $0(0)$ & $18(78)$ \\
\hline Range (mg/dl) & $0 \cdot 0-9 \cdot 3$ & $4 \cdot 2$ & 0 & $0.0-0.19$ & $0.0-0.51$ & $0 \cdot 0-4 \cdot 2$ & 0 & $0 \cdot 0-1 \cdot 8$ \\
\hline Mean (mg/dl) & 0.95 & $0 \cdot 18$ & 0 & 0.02 & 0.06 & 0.50 & 0 & 0.32 \\
\hline
\end{tabular}

SI conversion: $\mathrm{mg} / \mathrm{dl} \times 10=\mathrm{mg} / \mathrm{l}$.

were uniformly absent. Nonseptic inflammatory fluids had a similar volatile SCFA pattern to that seen in the noninflammatory group except for fluids from 6 patients, including 2 with rheumatoid arthritis, 2 with unspecified polyarthritis, 1 with Reiter's syndrome, and 1 with inflammatory bowel disease that did contain propanoic acid. A majority of septic fluids contained pentanoic and hexanoic acids. A minority of synovial fluids had acetic, propanoic, butanoic, or 3-methyl butanoic acids in no characteristic pattern.

Fig. 1 shows the concentration of pentanoic acid in the $\mathbf{8 0}$ synovial fluids studied. The mean concentration of pentanoic acid in septic synovial fluids was no different from the mean concentration in the nonseptic inflammatory group and the nonseptic noninflammatory group $(0 \cdot 50 \mathrm{mg} / \mathrm{dl} \pm 0 \cdot 19,0 \cdot 68 \mathrm{mg} / \mathrm{dl}$

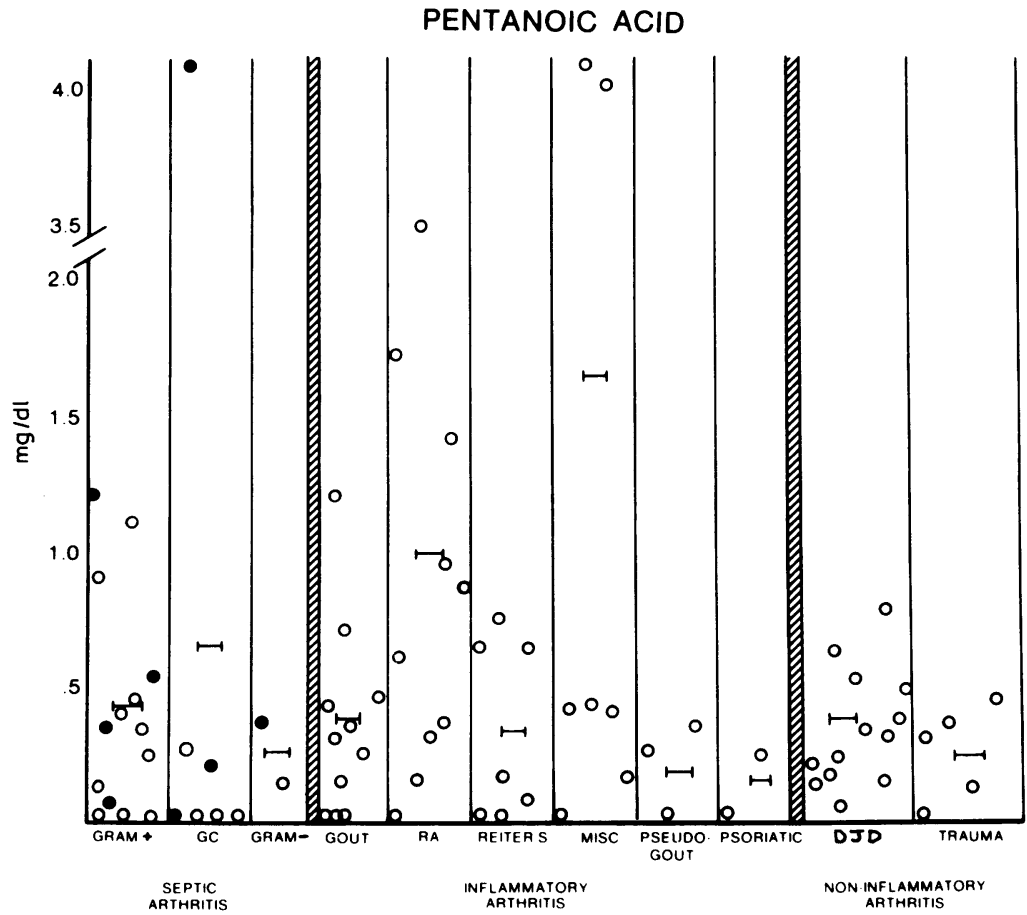

Fig. 1 Pentanoic acid concentrations for 80 synovial fluids. Open circles (O) represent synovial fluids taken from patients prior to antibiotic therapy. Closed circles (O) represent synovial fluids taken from patients during antibiotic therapy. Mean concentrations are designated by horizontal lines (_- ). 
HEXANOIC ACID

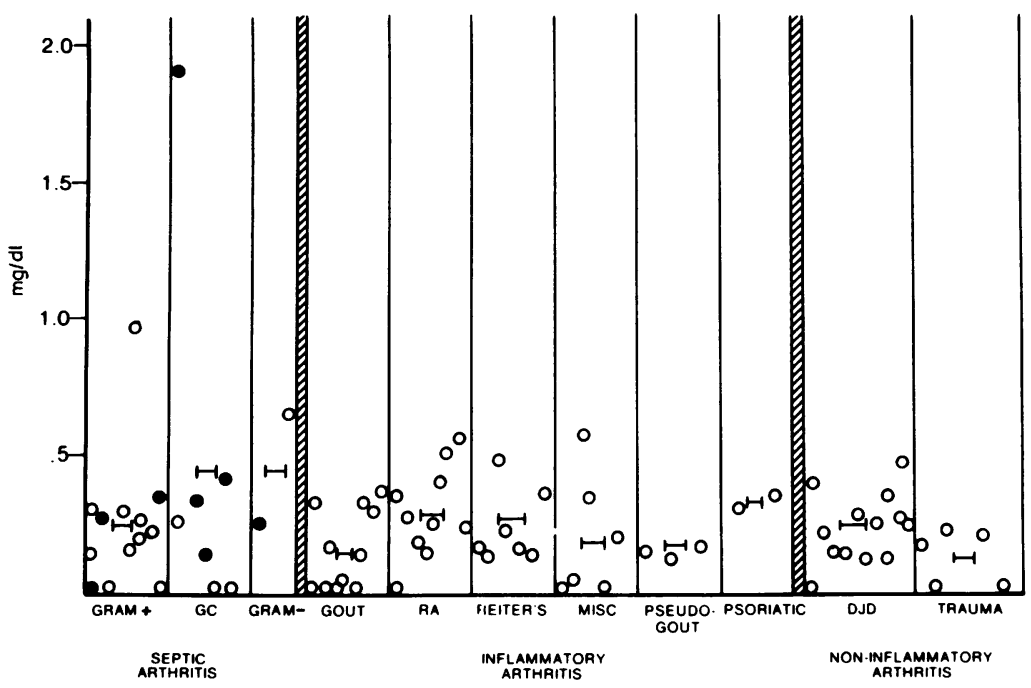

Fig. 2 Hexanoic acid concentrations for 80 synovial fluids. Open circles (O) represent synovial fluids taken from patients prior to antibiotic therapy. Closed circles (O) represent synovial fluids taken from patients during antibiotic therapy. Mean concentrations are designated by horizontal lines ( - ). $\pm 0 \cdot 15,0.33 \mathrm{mg} / \mathrm{dl} \pm 0 \cdot 21 ; \mathrm{p}$ not significant). (SI conversion: $\mathrm{mg} / \mathrm{dl} \times 10=\mathrm{mg} / \mathrm{l}$ ).

Fig. 2 shows the concentration of hexanoic acid in the 80 synovial fluids studied. The mean concentration of hexanoic acid in septic synovial fluids was no different from the mean concentration in the nonseptic inflammatory group or the nonseptic noninflammatory group $(0.32 \mathrm{mg} / \mathrm{dl} \pm 0.05,0.22 \mathrm{mg} / \mathrm{dl}$ $\pm 0 \cdot 04,0 \cdot 20 \mathrm{mg} / \mathrm{dl} \pm 0 \cdot 06 ; \mathrm{p}$ not significant).

Acetic acid was detected in 5 synovial fluids from patients with Gram-positive septic arthritis. Three fluids were from patients with Staphylococcus aureus septic arthritis, 1 was from a patient with a Streptococcus pneumoniae infection, and 1 was from a patient with Gram-positive cocci on smear but a negative culture. The 3 fluids which had detectable acetic acid in the nonseptic inflammatory group included 1 each from patients with pseudogout, inflammatory bowel disease, and unspecified polyarthritis. The 3 fluids with detectable acetic acid in the nonseptic, noninflammatory group included 2 from patients with osteoarthritis and 1 from a patient with trauma.

In addition to acetic acid, butanoic acid was detected in 1, propanoic acid in 1, and 3-methyl butanoic acid in 3 fluids from patients with staphylococcal septic arthritis. The patient with a Proteus morganii infection had butanoic acid as well as pentanoic acid and hexanoic acids. In 1 patient with a gonococal infection butanoic acid was detected in addition to pentanoic acid and hexanoic acid.

In the septic group 3 synovial fluids had no detectable volatile SCFA, including 1 from a patient with a Str. pyogenes infection and 2 from patients with gonococcal infections.

\section{Discussion}

GLC is a rapid and sensitive method for detecting nonvolatile and volatile SCFA and other carboxilic acids in synovial fluid. Characteristic patterns of nonvolatile carboxilic acids in septic synovial fluid have been previously reported. ${ }^{4}$ Volatile SCFA have also been suggested as markers of septic synovial fluid in one study. ${ }^{5}$ In contrast to that study our study did not demonstrate qualitative or quantitative differences in volatile SCFA in synovial fluid from patients with septic, nonseptic inflammatory, or noninflammatory joint diseases.

Volatile SCFA have been detected in body fluids infected with anaerobic organisms. Ladas et al. ${ }^{7}$ reported the presence of acetic, propanoic, 2-methyl propanoic, butanoic, and 3-methyl butanoic acids in anaerobic infections of peritoneal or abscess fluids from 11 patients. In the same study aerobic infections with Staph aureus, Escherichia coli, Pseudomonas aeruginosa, or Klebseilla pneumoniae resulted in 
detectable acetic, lactic, and succinic acids only. Phillipset al. ${ }^{8}$ reported similar volatile SCFA findings in anaerobic infections in peritoneal, pleural, and abscess fluids from 24 patients.

Volatile SCFA determinations of 147 blood cultures infected by aerobic bacteria was reported by Wust. ${ }^{9}$ Smaller concentrations of propanoic, 2-methyl propanoic, butanoic, and 3-methyl butanoic acids were detected in blood cultures infected with a wider variety of Gram-positive and Gramnegative aerobic organisms than reported with anaerobic bacteria. Phillips et al. ${ }^{8}$ reported that the only SCFA detected in 5 of 16 peritoneal, abscess, or synovial fluids infected with aerobic bacteria was acetic acid. Staph aureus caused the infection in all 5 patients with detectable acetic acid.

Seifert et al. ${ }^{2}$ reported the detection of acetic acid in addition to increased concentrations of lactic acid in 5 synovial fluids from patients with culture-proved septic arthritis, including 3 with Staph aureus infection. Brook et al. ${ }^{5}$ reported significant increases in the mean value of volatile SCFA, presumably pentanoic and hexanoic acids, in 44 synovial fluids from patients with septic arthritis compared with 42 with inflammatory and 8 with noninflammatory arthritis. Patients with gonococcal arthritis had increased synovial fluid concentrations of pentanoic and hexanoic acids with no increase in lactic acid. Other volatile SCFA were detected with no specific pattern. Thus these data suggested that an increased lactic acid concentration was helpful in identifying patients with nongonococcal septic arthritis, while increased pentanoic and hexanoic acid concentrations identified patients with gonococcal arthritis.

Our study of 80 synovial fluids found no statistically significant difference in the mean concentration of pentanoic or hexanoic acids in septic, nonseptic inflammatory, and noninflammatory groups. In the septic group pentanoic and hexanoic acid concentrations were similar for synovial fluids infected with Gram-positive, nongonococcal Gram-negative, or gonococcal organisms. The difference between our results and those reported by Brook et al. ${ }^{5}$ may be explained in part by differences in methodology. In our study absolute concentrations of fatty acids were compared with an internal standard which was added to each sample. This procedure assured accurate quantification of SCFA despite unexpected changes in assay conditions. Brook et al. $^{5}$ reported peak height in millimeters as the measurement of fatty acid concentration. No internal standard was used for absolute quantification of fatty acids. Injection size, oven temperature, and gas flow, among other factors, may affect peak height on the recorder.

While pentanoic and hexanoic acids were not detected in increased concentrations, acetic acid was found in 4 synovial fluids with culture-proved septic arthritis. Three of these 4 fluids were infected with Staph aureus; 3-methyl butanoic acid was found in the same 3 fluids infected with Staph aureus. Five synovial fluids infected with Staph aureus had no detectable acetic or 3-methyl butanoic acids. Six nonseptic fluids had acetic acid but none had detectable 3-methyl butanoic acid.

Organic acids detected in septic synovial fluid are products of host and bacterial carbohydrate and lipid metabolism. Lactic acid may be produced by synovial membrane in patients with inflammatory joint disease. ${ }^{10}{ }^{11}$ Lactic and acetic acids are end products of staphylococcal glucose catabolism in aerobic and anaerobic conditions. ${ }^{12}$ Fatty acids in the form of phospholipids are major constituents of host and bacterial membranes. Most bacterial fatty acids range in size from 12 to 18 carbons, but during their synthesis or degradation shorter chain fatty acids are produced. Gram-negative organisms typically contain saturated, unsaturated, and cyclopropane fatty acids. Gram-positive organisms contain saturated and branched fatty acids. ${ }^{13}$ Pentanoic and hexanoic acids detected in this study might be produced by the metabolism of host and bacterial straight-chain, saturated fatty acids. 3-Methyl butanoic acid might be produced by the metabolism of branched fatty acids from Gram-positive bacteria, such as Staph aureus. ${ }^{14}$

In summary, we found that septic synovial fluid could not be differentiated from nonseptic inflammatory or noninflammatory fluids by determinations of volatile SCFA. Nonvolatile carboxilic acids are more sensitive as diagnostic markers for septic arthritis, as previously reported. In patients with synovial fluid findings consistent with septic arthritis, including markedly raised leucocyte count, decreased glucose concentration, or detectable succinic acid, GLC analysis for detection of acetic and 3-methyl butanoic acids may be useful. The detection of these volatile SCFA in an infected synovial fluid may increase the suspicion that Staph aureus is the cause of the septic process.

We thank Dr Seth Lourie, Dr Werner Barth, and Dr Gary Ruppert for supplying synovial fluids, and Patricia Cleary of the George Washington University Biostatistics Center for statistical analysis of the data for this study. We also express our appreciation to Marc Roskelley for his expertise in the typing of the manuscript.

This work was supported in part by a grant from the Arthritis and Rheumatism Association of Metropolitan Washington and Biomedical Research Grant NIH 5-S07-RR-05359-18, Proj. 6-79.

\section{References}

1 Garcia-Kutzback A, Dismuke S E, Masi A T. Gonococcal arthritis: clinical features and results of penicillin therapy.J Rheumatol 1974; 1: 210-21. 
2 Seifert M H, Mathews J A, Phillips I, Gargan R A. Gas-liquid chromatography in diagnosis of pyogenic arthritis. $\mathrm{Br}$ Med $\mathrm{J}$ 1978; ti: 1402 .

3 Brook I, Reza M J, Bucknell K S, Bluestone R, Finegold S M. Synovial fluid lactic acid: a diagnostic aid in septic arthritis. Arthritis Rheum 1978; 21: 774-9.

4 Borenstein D G, Gibbs C A, Jacobs R P. Gas-liquid chromatographic analysis of synovial fluid: succinic acid and lactic acid as markers for septic arthritis. Arthritis Rheum 1982; 25: 947-53.

5 Brook I, Reza M J, Bucknell K S, Bluestone R, Finegold S M. Abnormalities in synovial fluid of patients with septic arthritis detected by gas-liquid chromatography. Ann Rheum Dis 1980; 39: 168-72.

6 Holdman L V, Cato E P, Moor W R C. Anaerobic laboratory manual. 4th ed. Blacksburg, Virginia: Virginia Polytechnic Institute and State University, 1977: 11-136.

7 Ladas S, Arapakis G, Malamou-Ladas H, Palikaris G, Aiseni A. Rapid diagnosis of anaerobic infections by gas-liquid chromatography.J Clin Pathol 1979; 32: 1163-7.
8 Phillips K D, Tearle P V, Willis A T. Rapid diagnosis of anaerobic infections by gas-liquid chromatography of clinical material. $J$ Clin Pathol 1979; 29: 428-32.

9 Wust J. Presumptive diagnosis of anaerobic bacteremia by gasliquid chromatograp hy of blood cultures.J Clin Microbiol 1977; 6: 586-90.

10 McCarty D J. Selected aspects of synovial membrane physiology. Arthritis Rheum 1974; 17: 289-96.

11 Treuhaft P S, McCarty D J. Synovial fluid pH, lactate, oxygen, and carbon dioxide partial pressures in various joint diseases. Arthritis Rheum 1971; 14: 475-84.

12 Theodore T S, Schade A L. Carbohydrate metabolism of ironrich and iron-poor Staphylococcus aureus. J Gen Microbiol 1965; 40: 385-95.

13 Shaw N. Lipid composition as a guide to the classification of bacteria. Adv Appl Microbiol 1974; 17: 63-108.

14 Lennarz W J. Lipid metabolism in the bacteria. Adv Lipid Res 1966; 4: 175-220.

\section{Book review}

Journal of Clinical Apheresis. Vol. 1. (Quarterly). Ed. C. Harold Mielke, Jr. Pp. 55. Subscription US \$81·00. Alan R. Liss: New York. 1982.

Connective Tissue Diseases. Vol. 1. (Half-yearly). Ed. Mario Giordano. US \$70•00. Idelson: Naples. 1982.

These 2 new journals are to my mind representative of certain persisting differences between American and European approaches to medicine. Clearly if you have pheresis hardware and a budget for it, it is important to stay up to date with benefits, safety, clinical indications, mechanisms, and technology. It is worth noting, however, that in California a State Senate Bill is being produced to exempt private apheresis clinics from regulations which require any new medical facility to prove that a need exists for it. The State Chapters of the Arthritis Foundation are opposing this Bill, as they regard the whole thing as premature commercialisation. If Goodpasture's syndrome were rather commoner perhaps a stronger case could be made. However, pheresis in all its form (leucapheresis, plasma exchange, etc.) is here to stay and must be evaluated. Millward's piece on the historical development of automated haemapheresis, from its beginnings in the Swedish dairy industry makes interesting reading. So does a report from the National Centre for Health Care pointing out the startling potential cost of widespread apheresis for rheumatoid arthritis (even it it does work: for Ivan Illich it would confirm 'high technology' medicine's conspiracy). Another uncontrolled study of 6 patients with lupus nephritis is also reported.

In his editor's foreword to the first number of Connective Tissue Diseases Professor Giordano states, 'essentially the spirit of this journal lies in considering the Connective Tissue as the protagonist of different pathologic events and therefore lies in proposing it as a point of inter-disciplinary encounter'. This journal seems to be aiming at those who feel that the present subspecialty organ-orientation impairs understanding of ubiquitous pathological processes. Two introductory articles by the editor and 3 on eosinophilic fasciitis, all in English, are followed in Italian by notes and reviews of articles in other journals and an appreciation of the late Professor Delbarre in French.

The 'apheresis' journal has an undoubted predetermined readership. Whether Connective Tissue Diseases has one also remains to be seen. With shrinking personal and library budgets I have my doubts.

M. L. SNAITH 